\title{
Virtual microfluidic traps, filters, channels and pumps using Marangoni flows
}

\author{
Amar S Basu ${ }^{1}$ and Yogesh B Gianchandani \\ Department of Electrical Engineering and Computer Science, University of Michigan, \\ Ann Arbor, MI, USA \\ E-mail: abasu@eng.wayne.edu
}

Received 22 May 2008, in final form 18 August 2008

Published 22 October 2008

Online at stacks.iop.org/JMM/18/115031

\begin{abstract}
This paper describes how Marangoni flows of various forms can be generated in thin liquid films for the purposes of microfluidic manipulation. Several microfluidic components, including traps, channels, filters and pumps, for manipulating aqueous droplets suspended in a film of oil on blank, unpatterned substrates are demonstrated. These are 'virtual' devices because they have no physical structure; they accomplish their function entirely by localized variations in surface tension (Marangoni flows) created in a non-contact manner by heat sources suspended just above the liquid surface. Various flow patterns can be engineered through the geometric design of the heat sources on size scales ranging from 10 to $1000 \mu \mathrm{m}$. A point source generates toroidal flows which can be used for droplet merging and mixing. Virtual channels and traps, emulated by linear and annular heat fluxes, respectively, demonstrate nearly $100 \%$ size selectivity for droplets ranging from 300 to $1000 \mu \mathrm{m}$. A source of heat flux that is parallel to the surface and is triangular with a $10^{\circ}$ taper serves as a linear pump, translating droplets of about the same size at speeds up to $200 \mu \mathrm{m} \mathrm{s}^{-1}$. The paper includes simulations that illuminate the working principle of the devices. Models show that Marangoni flows scale favorably to small length scales. By using microscale thermal devices delivering sharp temperature gradients, it is possible to generate $\mathrm{mm} \mathrm{s}^{-1}$ flow velocities with only small increases $\left(<1^{\circ}\right)$ in liquid temperature.
\end{abstract}

(Some figures in this article are in colour only in the electronic version)

\section{Introduction}

Marangoni flow, which is the movement of liquids due to surface tension gradients, is responsible for many common phenomena. These include, for example, the dispersion of oils in dishwater upon the addition of detergent [1] and the rotating flow of particles below a candle wick [2]. At macroscopic length scales, the Marangoni effect is not a practical mechanism for fluidic actuation because surface forces are typically weak compared to pressure, inertial and other body force mechanisms. However, like other surface phenomena, it scales favorably to the micrometer regime, and the high ratio of surface area to volume present in microscale devices suggests that the Marangoni effect could be useful for microfluidic actuation.

\footnotetext{
1 Present address: Electrical and Computer Engineering Department, Wayne
} State University, 5050 Anthony Wayne Drive, Detroit, MI 48202, USA.
The traditional, 'macroscopic' approach for generating Marangoni flow is via isothermal heating. A thin liquid layer is heated uniformly from below, and the resulting flow is a pattern of hexagonal convection cells [3]. First observed by Benard in the late 1800s [4], this multicellular flow was originally thought to be due to natural convection, and was later attributed to the Marangoni effect by Brock and Pearson in the 1950s [5-7]. Since then, it has been the subject of several efforts to mathematically analyze fluid stability [3]. From a practical standpoint, however, the geometry and stability of the flow are very sensitive to the fluid container and the thermal boundary conditions. Therefore, isothermallygenerated Marangoni flows provide little opportunity for localized fluidic manipulation in the context of an automated system.

Another way to generate controlled Marangoni flow on a liquid film is to impose micrometer scale temperature 

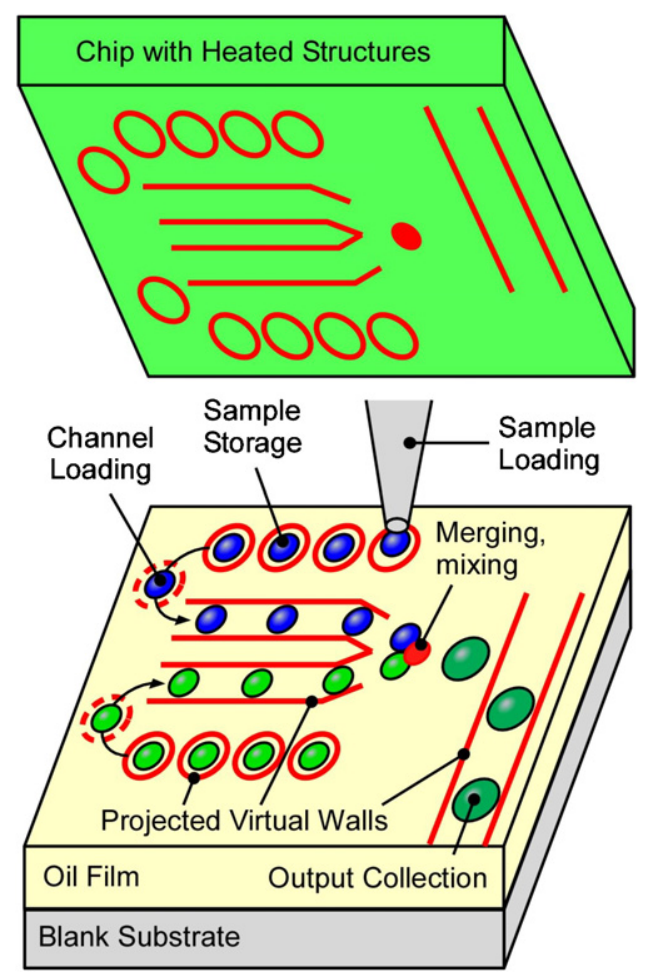

Figure 1. Concept of a contactless microdroplet manipulator based upon Marangoni flows. The flow is driven by heat sources of various geometries suspended above the oil layer. The projected heat fluxes (shown in red) generates flows which emulate droplet channels, reservoirs and mixers.

gradients on the surface, which can be done by suspending small heat sources just above the liquid surface [8]. This approach exploits the favorable scaling of surface forces and could provide several important advantages in practicality and efficiency, including flow localization and efficient energy coupling. These will be described later in the paper.

The primary benefit, and the focus of this paper, is the ability to engineer Marangoni flows. Using microscale heat sources of various geometries, it is possible to shape the surface temperature gradient and the resulting Marangoni flow. This effort shows that a number of useful flow patterns can be obtained in thin layers of oil using simple geometries, such as point heat sources, lines, rings and tapered shapes.

With respect to lab-on-a-chip systems, the purpose of these flows is to enable the manipulation of microdroplets in an oil layer. The flows demonstrated in this paper ${ }^{2}$ are capable of emulating the function of droplet traps, channels, filters and pumps. It is envisioned that these virtual components could be integrated into a noncontact microfluidic system driven only by heat fluxes (figure 1).

Section 2 outlines general theoretical and simulation models of Marangoni flow. Section 3 gives simulation results focusing on each geometry of flow: a point heat source, which generates toroidal flows useful for trapping particles and droplets; linear heat sources emulating virtual channels and filters; ring heat sources acting as single droplet traps; and tapered heat sources emulating a pump. Section 4 presents

\footnotetext{
2 Portions of this work have appeared in conference abstract form in [9-11].
}

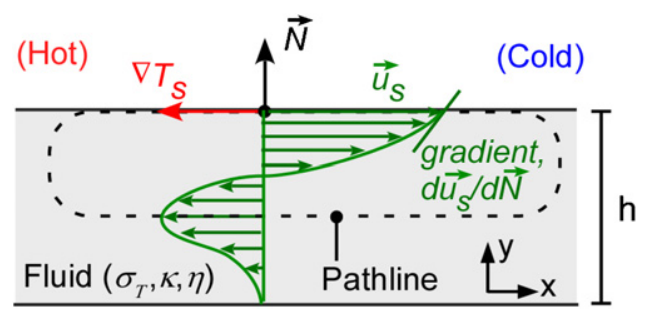

Figure 2. Theoretical formulation of Marangoni flow driven by a surface temperature gradient. In this figure showing the liquid cross section, it is assumed that the left side of the fluid layer is heated, forming a temperature gradient oriented toward the left. $\nabla T_{S}$ is the surface temperature gradient, $\vec{u}_{S}$ is the tangential surface velocity of the Marangoni flow and $\vec{N}$ is the surface normal vector. The parallel flow vectors illustrate the typical profile of surface and subsurface flow.

the corresponding experimental data for each type of flow, and Section 5 discusses design and experimental considerations.

\section{Theoretical model of marangoni flows}

Marangoni flows occur on a liquid-gas interface on which there exists a gradient in surface tension. The difference in interfacial shear stress drives flow tangential to the surface, directed from regions of low surface tension to high surface tension [3]. In this effort, the surface tension gradient is created by imposing a temperature gradient upon the interface. For most liquids, and for small temperature perturbations, surface tension decreases linearly with increasing temperature. The temperature coefficient of surface tension, $\sigma_{T}$, can be calculated using the Eötvös law, $\sigma_{T}=k_{\gamma} / V_{m}^{2 / 3}$, where $V_{m}$ is the molar volume of the liquid and $k_{\gamma}=2.1 \times 10^{-7} \mathrm{~J} \mathrm{~K}^{-1}$ for most liquids $[12,13]$. Thus, if a spatial temperature profile is imposed on the liquid surface, it will be accompanied by a corresponding surface tension gradient, and the resulting interfacial shear stress $\left(\tau_{s}\right)$ is proportional to the temperature gradient $\nabla T_{S}$. For a Newtonian fluid, the shear stress also determines the surface velocity gradient, resulting in a surface stress boundary condition which is the basis of Marangoni flows [14]:

$$
\tau_{s}=\mu \frac{\partial \vec{u}_{S}}{\partial \vec{N}}=-\sigma_{T} \nabla T_{S}
$$

In this equation, $\mu$ is the dynamic viscosity of the fluid, $\vec{u}_{S}$ is the tangential surface velocity vector and $\vec{N}$ is the surface normal vector (figure 2). The shear stress and flow vector are oriented from regions of low surface tension to high surface tension, opposite to the temperature gradient. The surface Marangoni flow, therefore, is directed away from the heat source. It is accompanied by subsurface flow, oriented in the opposite direction, which provides the return path needed to maintain fluid continuity. The depth at which the subsurface flow reaches maximum velocity, defined as the inversion depth, depends on the length scale of the flow and the depth of the liquid. Together, the surface and subsurface flows create a recirculating cell which is the basis for all microfluidic components to be discussed in this paper. 
The Marangoni flow resulting from a surface heat flux of arbitrary geometry and magnitude can be computed numerically using a thermal computational fluid dynamics (CFD) solver (FLUENT6, Fluent Corp., Lebanon, NH). The simulation model can be planar, axisymmetric, or a full threedimensional model as necessitated by the symmetry of the input heat flux. The software determines the Marangoni flows from the given heat flux using an iterative procedure which includes the following steps: (1) calculating the surface temperature profile gradient using a heat conduction simulation, (2) equating the surface temperature gradient to surface shear stress using equation (1), and (3) solving the Navier-Stokes and continuity equations with the shear stress as a boundary condition. The alteration in the temperature profile as a result of the flow is reflected in the following iteration, and the process repeats until both sets of equations converge. (This algorithm is standard in FLUENT's segregated solver [15].) For further information on the theoretical formulation, the reader is referred to [14, 16-18].

To model the heat transfer from the suspended heat source, a heat flux is applied at the fluid surface. In a twodimensional simulation, for example, a heat flux $q(x)$ is applied to the surface $y=h$, forcing a vertical temperature gradient $\mathrm{d} T / \mathrm{d} y=-q(x) / \kappa$ at the interface. The lateral temperature gradient resulting from the heating is equated to a surface stress boundary condition using equation (1). Next, according to the kinematic boundary condition, the normal velocity at the interface is set to zero. At the bottom surface of the fluid layer, an isothermal ambient temperature $T_{A}=300 \mathrm{~K}$ is specified, along with a no-slip boundary condition. The same condition is applied in the lateral far field boundaries. If axisymmetric, planar, or 3D symmetry are present in the model, then the appropriate symmetry boundary conditions apply at plane of symmetry. These conditions nullify the orthogonal flow velocities as well as the orthogonal field gradients. For example, in the case of a two-dimensional simulation, $u=\mathrm{d} v / \mathrm{d} x=\mathrm{d} T / \mathrm{d} x=0$.

Mineral oil is chosen as the carrier fluid due to its low thermal conductivity and low volatility. Material properties used in the simulations are as follows [17, 19]: $\rho=$ $886 \mathrm{~kg} \mathrm{~m}^{-3}, \mu=0.026 \mathrm{~Pa} \mathrm{~s}, k=0.12 \mathrm{~W} \mathrm{~K}^{-1}, C_{P}=$ $1700 \mathrm{~J} \mathrm{~kg}^{-1} \mathrm{~K}^{-1}$ and $\partial \sigma / \partial T=-16 \times 10^{-5} \mathrm{~N} \mathrm{~m}^{-1} \mathrm{~K}^{-1}$.

Simulations were carried out for each of the four heat flux geometries explored in this effort, including (1) a point heat source, (2) linear-shaped heat sources, (3) a ring-shaped heat source and (4) a tapered heat source. Each geometry represents a specific microfluidic component (see table 1), and each will be discussed in turn.

\section{Simulation results}

\subsection{Point heat source}

The point heat source, the most basic geometry, serves as a fluidic trap. The point source generates radial surface temperature gradients and Marangoni flow directed outward on the surface, and inward below. In three dimensions, the combined flow resembles a toroid (figure 3(a)) which can trap
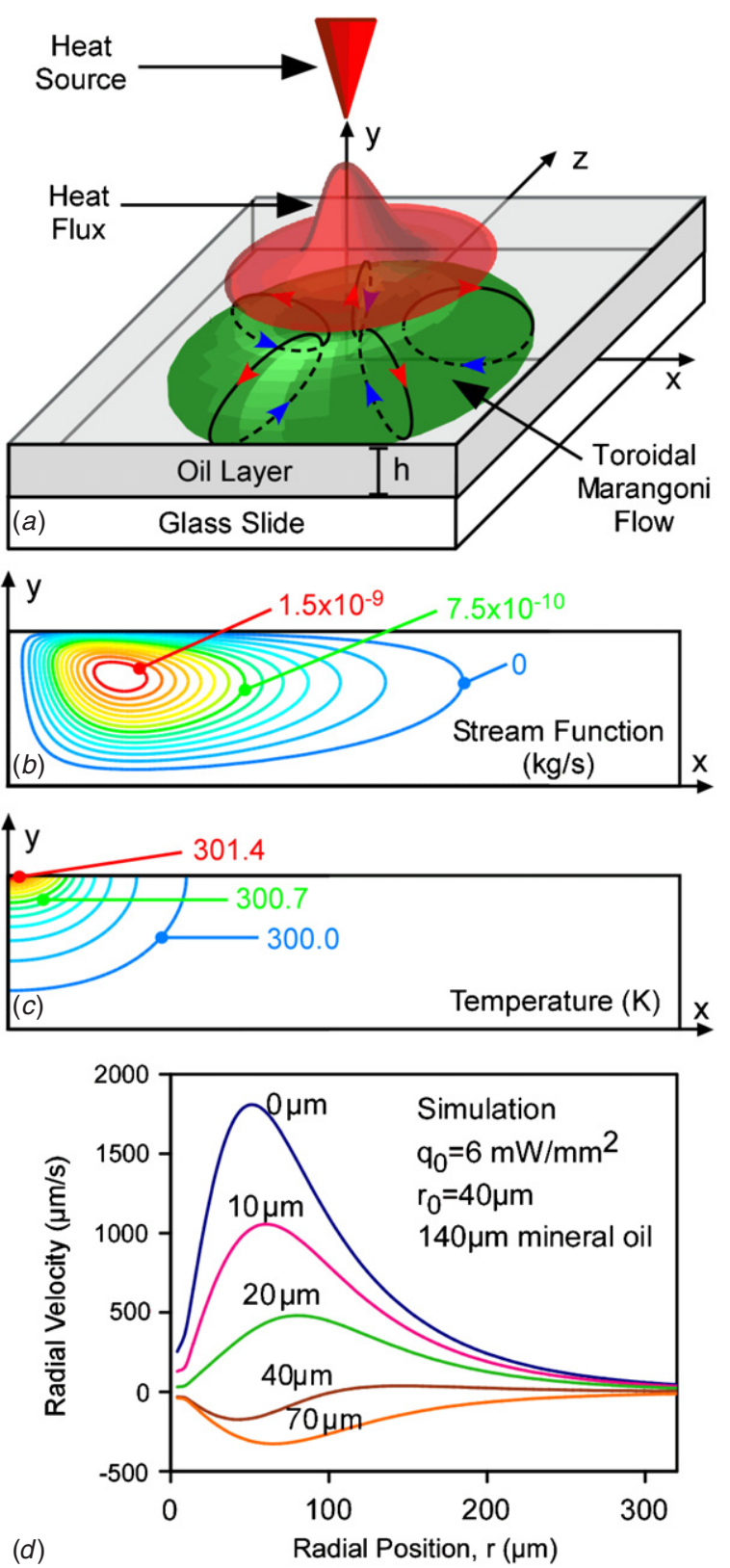

Figure 3. A microfluidic trap generated by a point heat source. (a) Schematic showing the suspended heat source, the projected Gaussian heat flux profile and the toroidal flow region in the oil layer. $(b)-(d)$ Thermal CFD simulation results showing flow resulting from a Gaussian heat flux with amplitude $q_{o}=$ $6 \mathrm{~mW} \mathrm{~mm}^{-2}$ and $r_{o}=40 \mu \mathrm{m}$ applied to a $140 \mu \mathrm{m}$ thick layer of mineral oil with surface tension coefficient $-0.16 \mathrm{mN} \mathrm{m}^{-1} \mathrm{~K}^{-1}$. (b) Contours of stream function, illustrating pathlines in the oil layer. The 20 contours are equally spaced between 0 and $1.5 \times$ $10^{-9} \mathrm{~kg} \mathrm{~s}^{-1}$. (c) Temperature profile in the oil layer, again with 20 equally spaced contours between $300 \mathrm{~K}$ and 301.4 K. (d) Spatial velocity profile at various depths in the liquid.

particles and droplets. Due to the axial symmetry, the point heat source is modeled in a two-dimensional geometry. The $140 \mu \mathrm{m}$ thick fluid layer is meshed with $5 \mu \mathrm{m}$ square elements, with refinements at the surface thermal boundary layer and the symmetry axis. A Gaussian heat flux $q(x)=q_{0} \exp \left(-x^{2} / r_{0}^{2}\right)$ is applied to the upper surface to represent the heating from the point heat source. The heat flux is given a peak amplitude 
Table 1. Summary of Marangoni flow resulting from various heat source geometries.

\begin{tabular}{lll}
\hline Shape & Function & Simulation \\
\hline Point heat source & Droplet collection, mixing, and rotation & Figures 3 and 4 \\
Linear heat sources (single and parallel) & Virtual droplet channel & Figures 5 and 6 \\
Annular & Single droplet trap & Figures $7(a)$ and $(b) \quad$ Figures $8-13$ \\
Tapered & Droplet pump & Figures $7(c)$ and $(d) \quad$ Figures $15(c)$ and $(d)$ \\
\hline
\end{tabular}

$q_{0}=6 \mathrm{~mW} \mathrm{~mm}^{-2}$ and a characteristic radius $r_{o}=40 \mu \mathrm{m}$, corresponding to $20 \mu \mathrm{W}$ total power at the surface. These values are chosen to correspond with flow velocities obtained in experiments. The liquid film is assumed to be mineral oil with the properties indicated above.

Simulation results of the point heat source are shown in figures $3(b)-(d)$. Contours of the stream function (figure $3(b)$ ), which represent the flow pathlines, convey the unicellular nature of the flow. If a particle or droplet is trapped in the flow, it continues to travel along the streamline unless upset by an external factor. The maximum surface temperature increase (figure $3(c)$ ) is $\Delta T_{\mathrm{MAX}}=1.5 \mathrm{~K}$ at the center, and the surface temperature drops off as a Gaussian function of the radial position. This is consistent with the dominance of conduction as the main heat transfer mechanism. (In contrast, the dominance of convection would lead to a wider spread in the temperature profiles.)

A typical simulated flow velocity profile at the surface and at several depths below the surface is shown in figure $3(d)$. It is notable that $1.7 \mathrm{~mm} \mathrm{~s}^{-1}$ maximum flow velocity is obtained with only a $1.5 \mathrm{~K}$ increase in surface temperature. The maximum velocity occurs about $40 \mu \mathrm{m}$ from the center of the heat source, corresponding to the location of the maximum temperature gradient. The radial velocity is positive at the liquid surface, reverses direction at approximately $30 \mu \mathrm{m}$ depth, attains a maximum negative velocity at $70 \mu \mathrm{m}$ depth and eventually diminishes to zero at the interface with the solid substrate. Simulations at various heat flux amplitudes show a linear dependence of surface velocity on heat flux-about $1.1 \mathrm{~mm} \mathrm{~s}^{-1}$ flow velocity is attained for every $1^{\circ}$ increase in surface temperature. The slope of this function is determined by the surface tension coefficient $\partial \sigma / \partial T$ and the surface temperature gradient $\partial T / \partial x$, the latter of which depends inversely on the thermal conductivity $\kappa$.

In this work, a microfabricated thermal probe serves as the point heat source. To model the interaction of the probe with the fluid surface, a full three-dimensional thermal fluid simulation is performed, which includes a $360 \times 120 \times 3 \mu \mathrm{m}$ thin film cantilever probe suspended in air, $15 \mu \mathrm{m}$ above a layer of mineral oil with thickness $200 \mu \mathrm{m}$. A heat generation condition of $1.5 \mathrm{~mW}$ is applied to a volume at the tip of the probe to emulate joule heating, resulting in a differential tip temperature of $20 \mathrm{~K}$. The resulting temperature contours (figure 4) show a sharp vertical gradient between the probe and the liquid surface, and a sharp lateral heat flux gradient due to the length scale. The surface heat flux results in a lateral temperature gradient that approximately resembles a point source, although the gradient across the probe is also reflected in the contours. Figures $4(b)$ and $(c)$ show a sharp decrease in surface temperature perturbation and surface
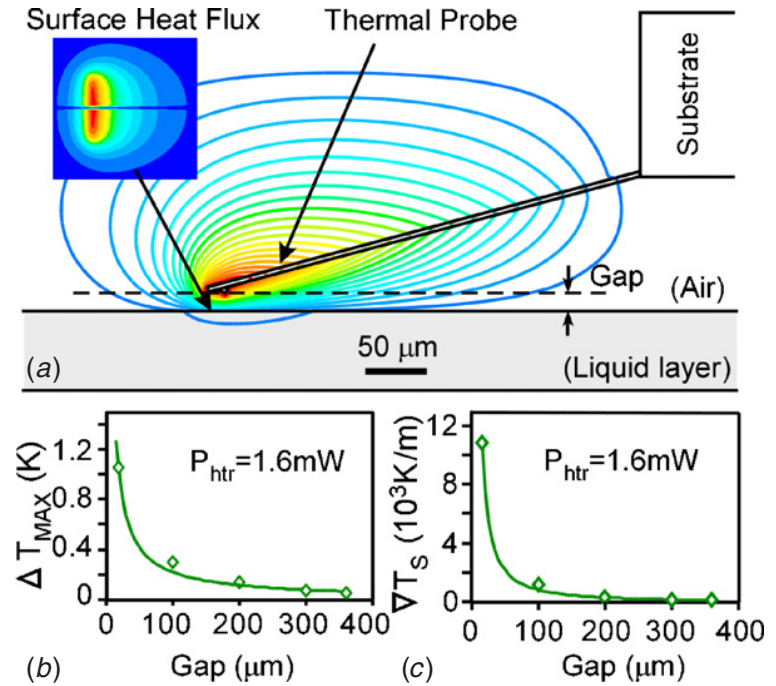

Figure 4. (a) Three-dimensional thermal fluid simulation showing the interaction of a microfabricated thermal probe held above a layer of mineral oil. The numerical value of the temperature contours depends on the applied power as shown in part $(b)$; however, the shapes of the contours do not change. The inset shows the qualitative surface heat flux resulting from the probe held at a $30^{\circ}$ angle. (b) Maximum temperature increase on the liquid surface versus separation between the probe and the liquid surface. (c) Maximum surface temperature gradient versus gap. In $(b),(c)$, the diamonds represent simulation results. The lines are a $1 / x$ best fit.

temperature gradient as the probe is moved away from the liquid surface. Both fall off as a function $1 / g$, where $g$ is the gap between the probe and the surface. This is due to the high thermal resistance of air, and it underscores the importance of maintaining a small air gap for maximum efficiency. The shear stress corresponding to a surface temperature gradient can be found by multiplying the temperature gradient by the surface tension coefficient $\partial \sigma / \partial T$. At a $15 \mu \mathrm{m}$ gap, the maximum shear stress is $1.6 \mathrm{~N} \mathrm{~m}^{-2}$, assuming $\partial \sigma / \partial T=-0.16 \mathrm{mN} \mathrm{m}^{-1}$. Another important aspect of this model is to investigate whether the convective air flow around the probe affects the Marangoni flow in the liquid. Simulation results show that flow velocities in air are several orders of magnitude less than the liquid Marangoni flow; thus, it is concluded that air flow has a negligible impact.

\subsection{Linear-shaped heat sources}

One level of complexity above the point heat sources are the linear heat sources, which emulate virtual microfluidic channels. Virtual channels collect droplets within a specific size range and confine them within their boundaries. One way to form a virtual channel is by using two parallel heat 


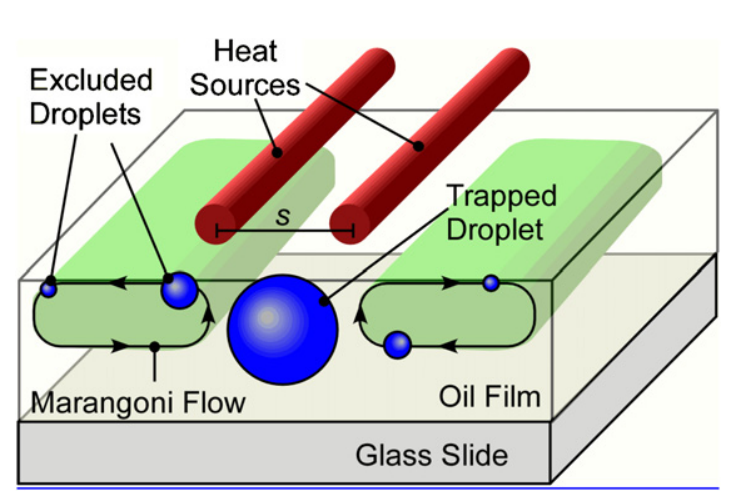

Figure 5. Virtual droplet channels generated by parallel, linear heat sources. The channel boundaries are defined by the heat flux projected by two heated wires with separation $s$ held parallel to the liquid surface. Target-sized droplets are pulled into the channel by the subsurface flows; others are excluded. Marangoni flows are shown in green and with arrows.

sources separated by a spacing $s$. When placed above a layer of oil, the sources project two linear heat fluxes on the liquid surface which define the boundaries of the channel (figure 5). The range of droplets captured within the channel is a function of the spacing, $s$. Target-sized droplets are pulled into the channel, while smaller droplets are pushed out by the surface flows. The mechanism of operation is shown using threedimensional CFD simulations (figure 6). In the simulation, a $5 \times 5 \times 0.2 \mathrm{~mm}$ fluid layer is meshed with $10 \mu \mathrm{m}$ square elements. The heat flux applied to fluid surface consists of a pair of one-dimensional Gaussian functions along the horizontal axis, with amplitude $q_{0}=6 \mathrm{~mW} \mathrm{~mm}^{-2}$, characteristic radius $r_{0}=40 \mu \mathrm{m}$ and a spacing of $900 \mu \mathrm{m}$ between the peaks. This results in two parallel peaks in surface temperature (figures $6(a)$ and $(c)$ ) as would be expected from two linear sources. The resulting Marangoni flow is comprised of two circulating cells on opposite sides of the heat sources, and a relatively still region between them. Surface flows are directed away from the virtual channel, and subsurface flows are directed toward it. A droplet of appropriate size is pulled into the channel by the subsurface flows, which apply a lateral force to the center of mass of the droplet. The surface flows, meanwhile, apply only a tangential force on the top side of the droplet and, therefore, do not cause lateral movement. In contrast, a smaller droplet, whose center of mass is closer to the surface, is pushed out of the channel by the surface flows and is, therefore, excluded. The distinction between 'large' and 'small' droplets is a function of $s$.

Another way to create a virtual channel is with a single line heat source instead of two parallel sources (figures $6(b)$ and $(d)$ ). In this case, the channel size and the droplet sizeselectivity are defined by the width of the heat source $(w)$ rather than the spacing $s$. Simulation conditions are identical to the previous, except that the applied surface heat flux includes only a single peak, and the characteristic radius is increased $\left(r_{o}=175 \mu \mathrm{m}\right)$ to represent a wider heat source. Simulation results illustrate the differences in flow patterns of the double and single line heat sources. The recirculating flows present inside the double line channel do not appear in the single line version; however, these internal flows do not affect the trapping capability of the channel. The double line heat source offers some flexibility in that the virtual channel region can be easily tuned wider than the single line channel for trapping larger droplets. It is notable that, in both cases, up to $4 \mathrm{~mm} \mathrm{~s}^{-1}$ surface velocities can be obtained with $<6 \mathrm{~K}$ change in surface temperature, again illustrating the potential efficiency of Marangoni flows. The subsurface flows are only about onethird of the velocity of the surface flows, but they are sufficient to pull in selected droplets.

\subsection{Annular heat source}

A single droplet trap can be implemented using an annular heat source (figures $7(a)$ and $(b)$ ). Compared to the parallel

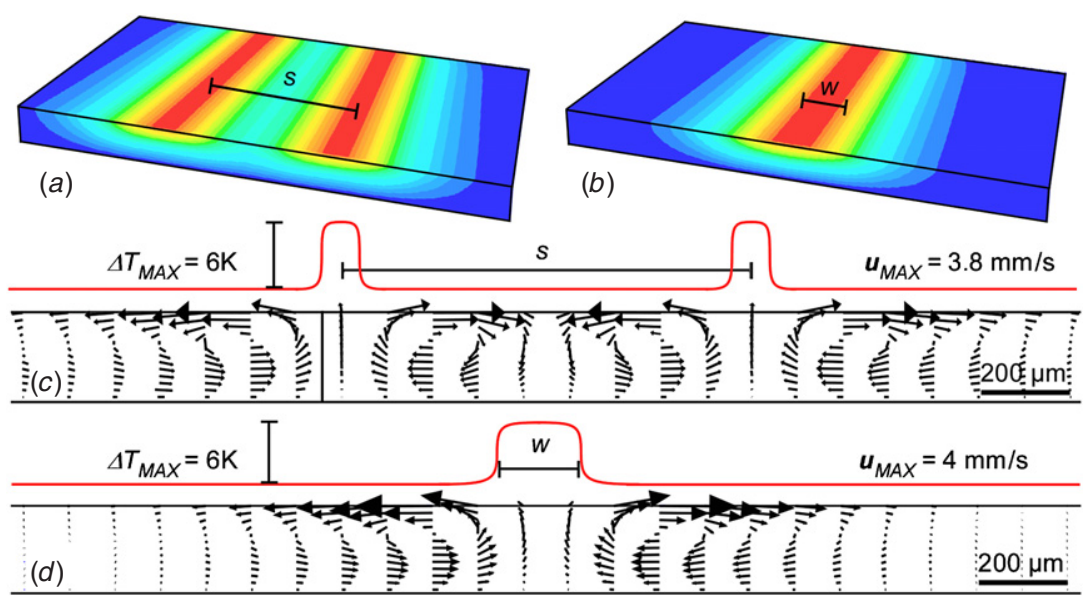

Figure 6. Simulations of a virtual channels formed by either two parallel heat fluxes with spacing $s$, or, alternatively, a single linear source with width $w$. (a), (c) show the temperature profile and flow vectors for two parallel linear heat sources with a spacing $s=900 \mu \mathrm{m}$. $(b),(d)$ show the same for a single line heat source with width $w=350 \mu \mathrm{m}$. In each case, surface temperature profiles are drawn above the flow vectors. Simulations assume a $200 \mu \mathrm{m}$ thick mineral oil layer with a surface tension coefficient of $-0.16 \mathrm{mN} \mathrm{m}^{-1} \mathrm{~K}^{-1}$. Heat flux amplitudes are Gaussian with amplitude $6 \mathrm{~mW} \mathrm{~mm}^{-2}$ and radius $40 \mu \mathrm{m}$. $u_{\mathrm{MAX}}$ is the maximum horizontal flow velocity, and $\Delta T_{\mathrm{MAX}}$ is the maximum surface temperature change. 
heat sources, the cross section of the flow vectors are identical; however, when viewed from above, the flow pattern is axiallysymmetric about the heat source. With respect to droplet manipulation, the primary difference between this component and the channel is that the droplet can be confined in both lateral dimensions. The trap is useful as a reservoir for holding single droplets, and for moving droplets if the heat source is translated laterally. On a broader note, a particularly interesting aspect of this flow pattern is the isolation of the fluid region within the confines of the ring. Simulations and experimental results show that the fluid within the ring boundary circulates independently and does not flow into the outer bulk fluid. In a sense, the inner and bulk fluid are doubly separated by two walls of co-rotating flows. This suggests that a ring heat source may have potential use as a fluid isolator outside the context of droplet systems.

In the simulation model, the heat flux profile is an axisymmetric ring shape, the cross section of which consists of Gaussian peaks at a radius of $300 \mu \mathrm{m}$. The amplitude and characteristic width of the Gaussian profile is maintained at the same values as prior simulations $\left(q_{0}=6 \mathrm{~mW} \mathrm{~mm}^{-2}\right.$ and $\left.r_{o}=40 \mu \mathrm{m}\right)$. Figure 7(b) shows the surface temperature profile $\left(\Delta T_{\mathrm{MAX}}=6 \mathrm{~K}\right)$ and flow resulting from a ring-shaped heat source in a $200 \mu \mathrm{m}$ thick layer of mineral oil. For clarity, the arrows show only the subsurface flows (140 $\mu \mathrm{m}$ beneath the surface) since they are responsible for droplet trapping. The flow can be divided into two distinct regions. Flow vectors outside the perimeter of the ring are oriented inward, and these flows push the droplet to the center. The maximum flow velocity in this region is $1.3 \mathrm{~mm} \mathrm{~s}^{-1}$. Inside the perimeter of the ring, the subsurface flows are oriented outward, reaching a peak velocity of $0.8 \mathrm{~mm} \mathrm{~s}^{-1}$; however, these flows do not affect the trapped droplet because the lateral forces they impart on a trapped droplet are symmetric and therefore cancel each other. As a result, target-sized droplets remain at the center of the ring. Like the earlier geometries, smaller droplets are rejected from the trap by the surface flows (not shown), which are oriented opposite the subsurface flows. Thus, the size exclusion capability applies to the annular geometry as well.

\subsection{Tapered heat source}

A fundamental component of any fluidic system is a pump. With noncontact Marangoni flows, pumping or actuation of droplets can be accomplished by two methods. The first is simply a consequence of the trapping: the heat source projecting a channel or a trap can be translated physically with respect to the substrate, and the trapped droplets follow it. However, droplet actuation can be also accomplished by steady-state, motionless heating provided by a tapered shape and angled spacing (figures $7(c)$ and $(d)$ ). The tapered geometry provides a triangular heat flux on the fluid surface and a temperature gradient oriented in the direction of increasing width. It can be simulated by applying a triangular heat flux with an amplitude of $6 \mathrm{~mW} \mathrm{~mm}^{-2}$ and a tip angle of $10^{\circ}$ on a $500 \mu \mathrm{m}$ square, $140 \mu \mathrm{m}$ thick layer of mineral oil. This results in a triangular temperature profile with a maximum temperature increase of $\Delta T_{\mathrm{MAX}}=5 \mathrm{~K}$ on the fluid surface.
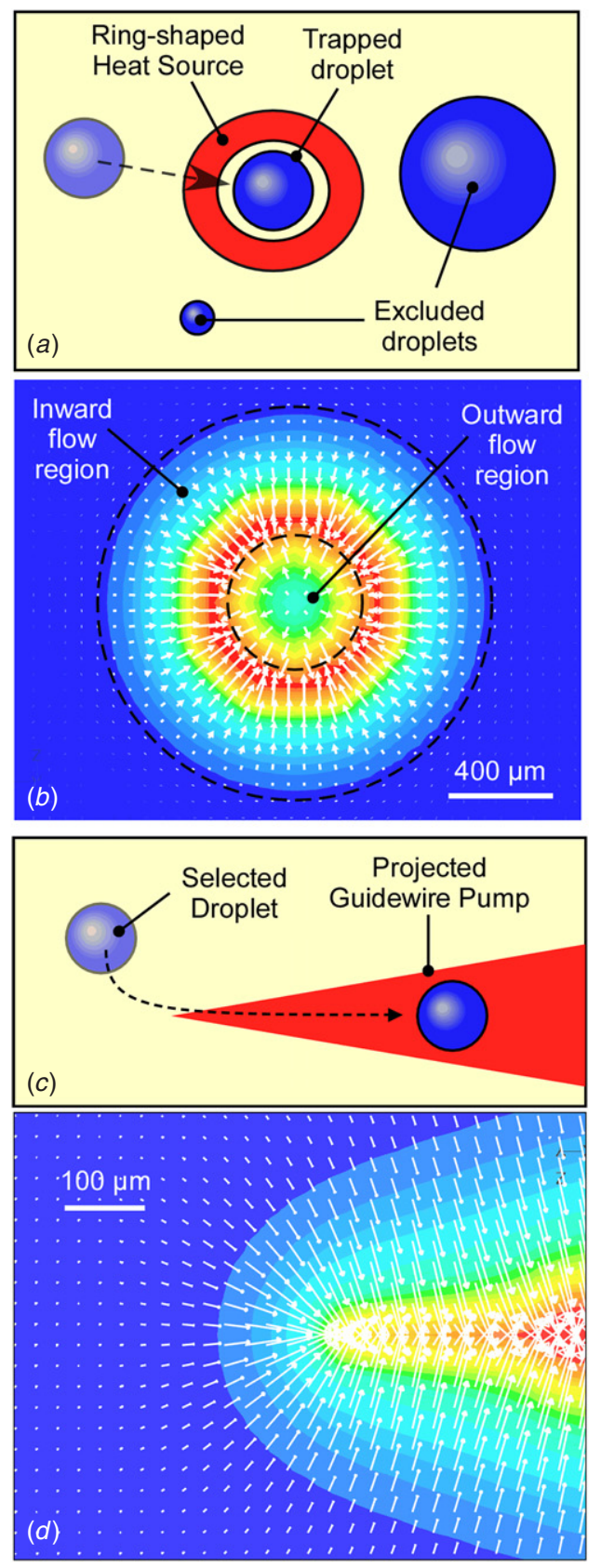

Figure 7. (a), (b) Single droplet trapping with an annular heat flux. (a) Schematic showing the annular heat flux projected on the surface, the trapped droplet and the exclusion of larger or smaller droplets. (b) CFD Simulation. The shaded contours represent the surface temperature gradient, with evenly spaced contours and a maximum temperature increase $\Delta T_{\mathrm{MAX}}=6 \mathrm{~K}$. White arrows represent the subsurface flow at $140 \mu \mathrm{m}$ depth beneath the surface. The two regions of flow are marked with dashed lines. $(c),(d)$ Guidewire pump, emulated by a tapered heat source. (c) Schematic showing the orientation of the triangular heat flux projected on the fluid surface, and a droplet being pulled in along its longitudinal axis. (d) CFD Simulation. Shaded contours represent the surface temperature gradient, and white arrows represent the subsurface flow (140 $\mu \mathrm{m}$ depth) which pushes the droplet from left to right.

The resulting subsurface flows (figure $7(d)$ ) are biased in the direction of increasing taper, and can pull appropriately sized droplets along the length of the guidewire. The maximum 


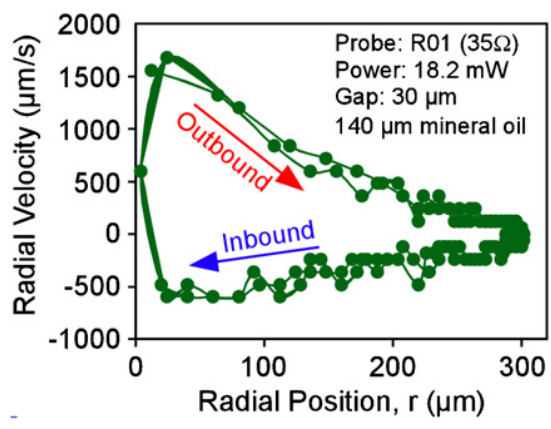

Figure 8. Experimental velocity profile of toroidal flow in $140 \mu \mathrm{m}$ thick mineral oil obtained with a thermal probe and the experimental conditions shown in the figure.

subsurface flow velocity of $1.5 \mathrm{~mm} \mathrm{~s}^{-1}$ can be found near the tip of the guidewire. The surface flows (not shown) have velocities roughly $3 \times$ larger than the subsurface flows and are biased in the opposite direction. As before, the surface flows affect only the small droplets, rejecting them from the guidewire.

\section{Experimental results}

\subsection{Point heat source}

Marangoni flows from point heat sources were obtained with a micromachined polyimide-shank thermal probe [20] which provides high thermal efficiency, sharp temperature gradients and controllable temperatures. The probe consists of a $40 \times$ $360 \times 3.5 \mu \mathrm{m}$ polyimide cantilever with a thin film heater near the tip which provides control of the probe power and temperature. The probe was mounted on a motorized micromanipulator stage (MP-285, Sutter Instruments, Novato, CA) and suspended above a $140 \mu \mathrm{m}$ thick layer of mineral oil (Mineral Oil USP, Rite Aid Corp., Harrisburg, PA). The thickness of the oil was measured optically. Flow velocities were measured at input powers ranging from 5 to $20 \mathrm{~mW}$ and at air gaps ranging from 20 to $320 \mu \mathrm{m}$. Weed pollen $(25 \mu \mathrm{m}$ diameter, Sigma-Aldrich, St Louis, MO) was immersed in mineral oil to serve as tracer particles in characterizing the flow, and also for demonstrating particle collection. Particle displacements were tracked using a CCD video camera (DXC-107, Sony USA, Culver City, CA) mounted on a stereomicroscope (SZX12, Olympus Corp, Center Valley, PA). Video clips were analyzed frame by frame to determine the position of a selected particle at $1 / 30 \mathrm{~s}$ intervals. Velocities were obtained by dividing the displacement over the time interval.

Experimental flow velocities are shown in figure 8. The spatial velocity profile, obtained by differentiating the displacement data in time, closely resembles the simulation results (figure $3(d)$ ) which used Gaussian heat fluxes with $q_{0}=$ $6 \mathrm{~mW} \mathrm{~mm}{ }^{-2}$ heat fluxes and $r_{0}=40 \mu \mathrm{m}$. The probe width itself is about $40 \mu \mathrm{m}$, showing reasonable correlation with the simulation. Flow velocities reach a maximum of $1.7 \mathrm{~mm} \mathrm{~s}^{-1}$. Although it is not feasible to accurately measure the temperature profile, the $1.5 \mathrm{~K}$ surface temperature elevation
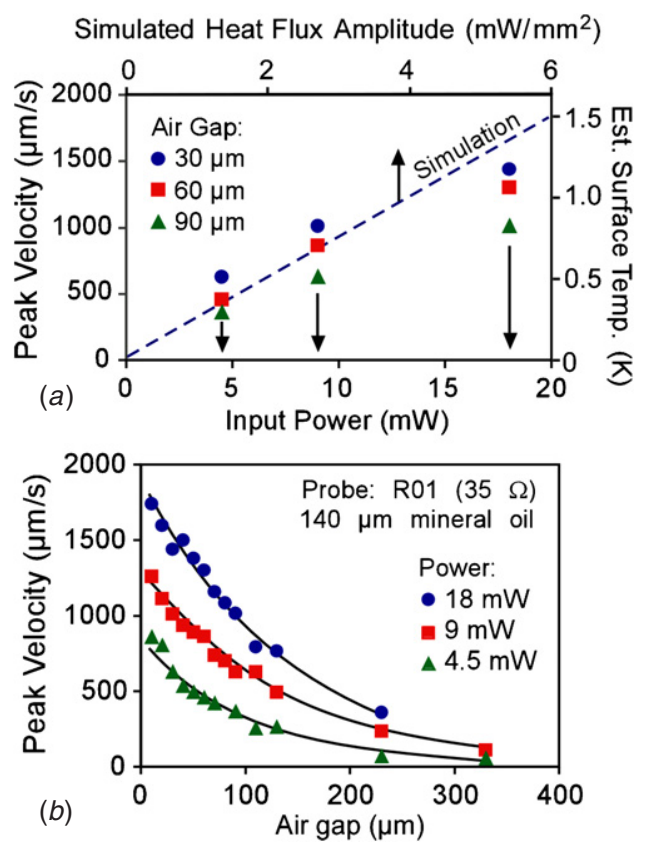

Figure 9. Control of flow velocities with toroidal flows generated by a microfabricated point heat source. (a) Flow velocity versus input power to the thermal probe plotted for three different separation distances. Also shown are simulated flow velocities as a function of input power. In the simulation, a Gaussian heat flux of increasing amplitude was applied to a $2 \mathrm{D}$, axisymmetric fluid layer. (b) Experimental flow velocity versus separation with experimental conditions as shown. A $140 \mu \mathrm{m}$ layer of mineral oil was used in all experiments and simulations.

found in simulations suggests that Marangoni actuation requires only small increases in surface temperature in order to function.

Figure 9(a) demonstrates the linear relationship between heat flux and flow velocity found in both simulations and experimental results. The linear offset in the experimental data was the result of an inadvertent photothermal effect: due to the high thermal isolation provided by the polyimide cantilevers, the microscope lamp heated the probe tip enough to create a non-zero velocity even when no electrical current was applied to the probe. Nevertheless, it can be seen that velocities scale approximately linearly with increasing power, and decrease exponentially with increasing separation between the probe and the liquid surface (figure $9(b)$ ).

Localization of the flow cell is an important parameter needed to selectively trap particles. Figure 8 shows that the flow cell is confined to an approximately $300 \mu \mathrm{m}$ radius from the center of the heat source. However, the radial extent of the flow can be made smaller by using a thinner fluid layer. It should be noted that flow velocities diminish in a thinner layer due to increased viscous forces between the flow layers (data not shown). Simulations and experimental results of the radial extent of flow versus fluid thickness show reasonable agreement (figure 10). In the simulation, an axisymmetric Gaussian heat flux with $q_{0}=6 \mathrm{~mW} \mathrm{~mm}^{-2}$ and radius $r_{0}=$ $50 \mu \mathrm{m}$ was applied to a mineral oil layer. In the experimental data, a $40 \times 360 \times 3.5 \mu \mathrm{m}$ probe with $18 \mathrm{~mW}$ applied power was placed approximately $100 \mu \mathrm{m}$ above the mineral oil layer. 


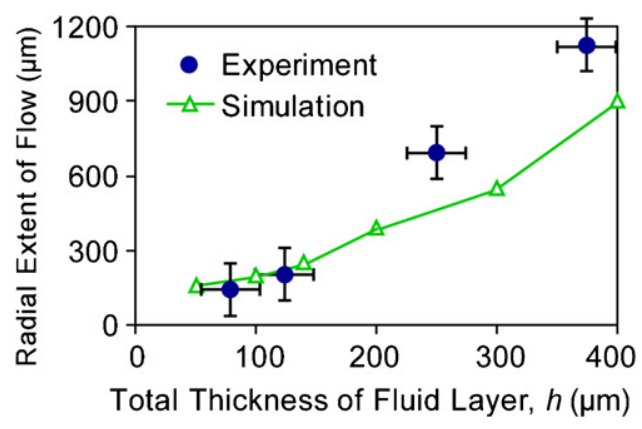

Figure 10. Effect of fluid layer thickness on the size of the flow cell.
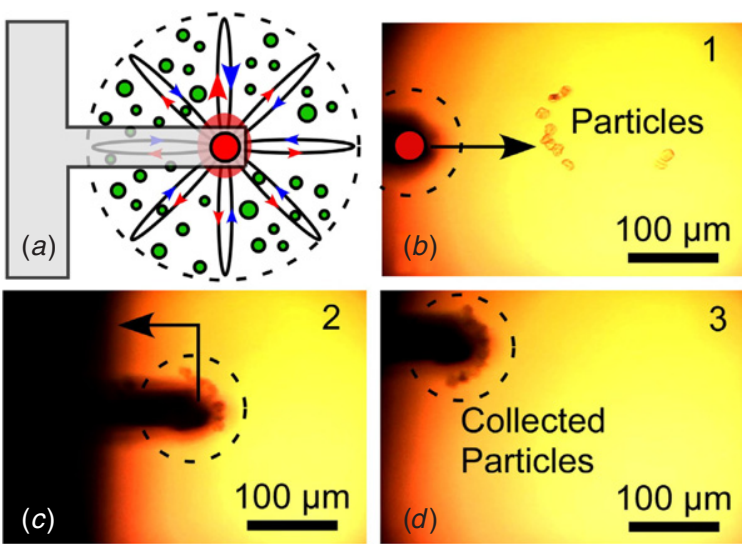

Figure 11. Localized particle collection using toroidal flows. (a) Schematic showing the heat source (red circle) connected to an $X Y$ scanning stage, toroidal flow patterns and particles (green circles) trapped within the circular collection region. $(b)-(d)$ Collection of $25 \mu \mathrm{m}$ weed pollen. (b) The heat source is first advanced toward the particles. (c) When the heat source reaches the area above the particles, the particles are swept into the vortex and trapped. $(d)$ The trapped particles are moved to a new location and released by turning off the heater.

Highly localized Marangoni flows can be useful for trapping and manipulating particles. For example, the thermal probe can be suspended above the liquid sample on an $X Y$ scanning stage so that the collection region can be shifted laterally (figure 11). In this demonstration, a thin fluid layer $(80 \mu \mathrm{m})$ is used to minimize the radius of the collection region and a low viscosity dielectric liquid (EDM185, Commonwealth Oil, Harrow ON) is chosen to help offset the increased shear that occurs in thin liquid layers. Figures $11(b)-(d)$ illustrate the harvesting of $25 \mu \mathrm{m}$ diameter weed pollen with a localized collection region of $50 \mu \mathrm{m}$ radius. In this experiment, the pollen particles were immersed in the $80 \mu \mathrm{m}$ thick dielectric oil, and an $18 \mathrm{~mW}$ heat source was held $50 \mu \mathrm{m}$ above the oil surface and scanned as indicated. The micrographs show that as the heat source is scanned laterally, the particles are swept into the flow cell and transported with it. Particles are released by turning off the heat source. The notable aspect of this approach is that the heat source does not physically contact the liquid or the particles.

By using the same approach, it is possible to collect and merge microdroplets with radii ranging from 5 to $100 \mu \mathrm{m}$. The ability to merge discrete droplets is important in microdroplet systems, as it allows reagents to be mixed at
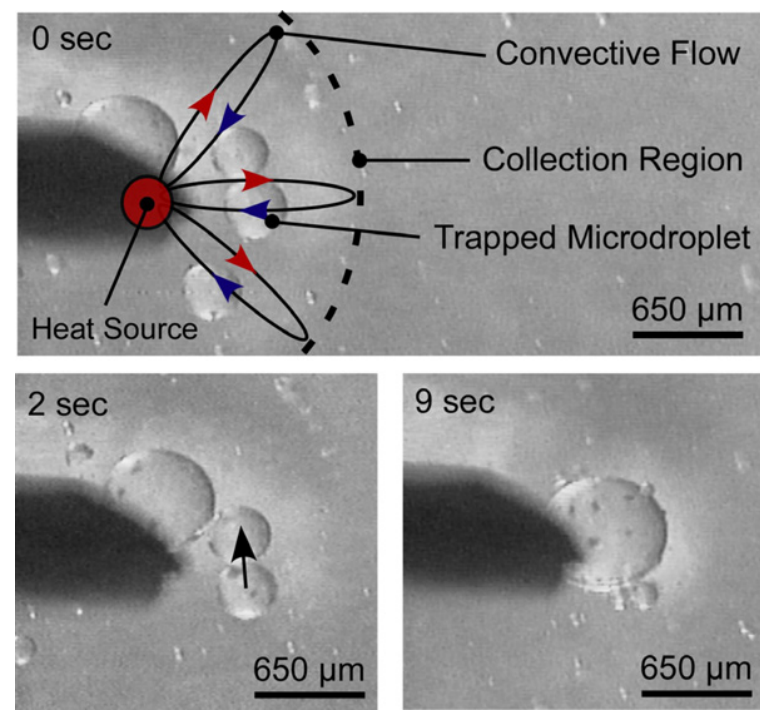

Figure 12. Droplet merging of four droplets with $(\phi=200$ $400 \mu \mathrm{m}) .(0 \mathrm{~s})$ The droplets are trapped in the self-circulating convective flow. $(2 s-9 \mathrm{~s})$ The droplets merge together, one by one, eventually forming a single, $\phi=600 \mu \mathrm{m}$ droplet.

millisecond time scales, fast enough to study chemical kinetics [21]. When brought in contact with one another, aqueous droplets spontaneously merge unless treated with a stabilizing surfactant. To demonstrate droplet collection and merging, a $650 \mu \mathrm{m}$ diameter, $350 \mathrm{~K}\left(77^{\circ} \mathrm{C}\right)$ wire tip was suspended $<100 \mu \mathrm{m}$ above mineral oil which contained droplets with diameters 200-400 $\mu \mathrm{m}$. The heat source collects droplets in the regions over which it is scanned. Droplets trapped in the flow eventually collide and merge with one another (figure 12). By scanning the heat source over a large area, many droplets can be collected and merged in this manner.

A third interesting capability of a point heat source is the ability to rotate and mix individual droplets. In general, when the particles and/or droplets immersed in the oil are small compared to the cross-sectional height of the convective flow region, they follow the toroidal streamlines. However, when the height of the flow region is approximately the same as the droplet diameter, the recirculating currents can rotate and mix the droplet in various patterns depending on the size of the heated tip (figure 13). In the case where the tip diameter is approximately the same size as the droplet, the Marangoni flows can rotate the droplet at high speeds. For example, if a $650 \mu \mathrm{m}$ diameter heated tip ( $T \approx 310 \mathrm{~K}$ or $37{ }^{\circ} \mathrm{C}$ ) is placed near a droplet of the same size (figure 13(b)) in an $800 \mu \mathrm{m}$ thick mineral oil layer, single axis rotation is observed at rates up to $300 \mathrm{rpm}$. In the case where the tip diameter $(50 \mu \mathrm{m})$ is smaller than the droplet $(1000 \mu \mathrm{m})$, the flow pattern is instead composed of two vortices [9]. Both of these manifestations can be useful for micro mixing within a single droplet.

With the intent of demonstrating the simplicity and generality of droplet manipulation methods, the demonstrations of droplet merging, rotation and mixing were carried out simply with a heated metal tip rather than the microfabricated thermal probe. Although not as thermally efficient or as small as the microfabricated heat source, the use of simple metal structures demonstrates the versatility of this 


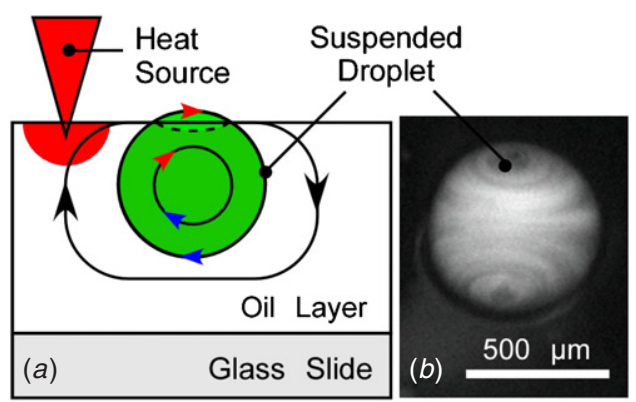

Figure 13. Droplet rotation and mixing. (a) Schematic showing Marangoni flows around a suspended droplet which can rotate the droplet. (b) Experimental results showing single axis rotation up to $300 \mu \mathrm{m} \mathrm{s}^{-1}$ obtained when a $650 \mu \mathrm{m}$ diameter tip is placed near a droplet of approx. the same dimensions. The tip temperature was $\approx 310 \mathrm{~K}\left(37^{\circ} \mathrm{C}\right)$. Flow patterns are visualized using immersed fluorescein particles and a $0.5 \mathrm{~s}$ CCD exposure with $490 \mathrm{~nm} /$ $500 \mathrm{~nm}$ excitation/emission filters.

technique. In the same spirit, simple metal structures are used to represent the ring, linear and tapered heat sources discussed in the remainder of this paper.

\subsection{Linear-shaped heat sources}

Virtual droplet channels, generated by linear heat sources, were experimentally verified by placing two parallel, cylindrical metal structures above a thin layer of phenylmethyl polysiloxane oil (Corning 550 Fluid, Dow Corning, Midland, MI). This particular oil was chosen for its high density (specific gravity $=1.07$ ), which causes the aqueous droplets to float on the oil surface. The suspension of the droplets is critical because droplet manipulations depend on the surface Marangoni flows. It also prevents contact between the droplet and the glass slide below. Droplets with diameters ranging from 20 to $2000 \mu \mathrm{m}$ were generated by pipetting $5 \mu \mathrm{L}$ of deionized water into the oil and stirring vigorously. The metal structures, with spacing $s=600 \mu \mathrm{m}$ and $s=900 \mu \mathrm{m}$, were located 200-400 $\mu \mathrm{m}$ above the oil surface and heated to approximately $380 \mathrm{~K}\left(107{ }^{\circ} \mathrm{C}\right)$. The size selectivity is demonstrated in figure 14 , which show that droplets within a specific size range move into the channel, while smaller droplets are actively removed by the Marangoni flows. Very large droplets are unaffected by the flows, and therefore are not actively recruited into the channel.

The ability to tailor the size selectivity of the channels is quantitatively shown in figure 14(b). A histogram of droplet capture versus droplet size is shown for the two channels with different spacings $(s)$. When $s=600 \mu \mathrm{m}$, the minimum diameter for entry into the channel is $250 \mu \mathrm{m}$, and when $s=$ $970 \mu \mathrm{m}$, the minimum diameter is $350 \mu \mathrm{m}$. A sharp transition can be seen in both cases, and nearly $100 \%$ exclusion of offsized droplets is shown. Therefore, these channels inherently provide a size-based filtration capability.

\subsection{Annular heat source}

Figures 15(a) and $(b)$ show experimental results for the annular heat source functioning as a droplet trap. A rectangular ring-
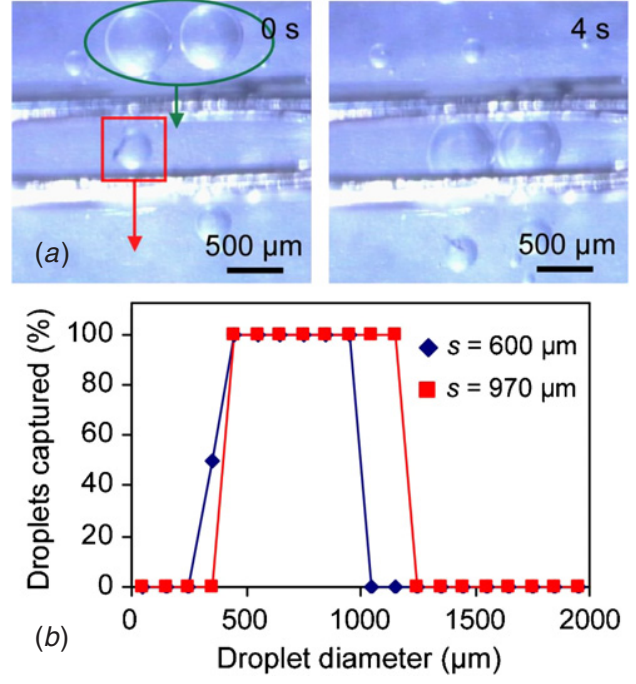

Figure 14. Size selective filtration of droplets in virtual channels. (a) Micrographs showing two $500 \mu \mathrm{m}$ diameter droplets (circle) entering the channel while a smaller one (square) is rejected. (b) Histogram showing the size selectivity of two droplet channels with $600 \mu \mathrm{m}$ and $970 \mu \mathrm{m}$ spacing. Droplet statistics were compiled over video clips spanning $40 \mathrm{~min}$. Bins are in $100 \mu \mathrm{m}$ increments.
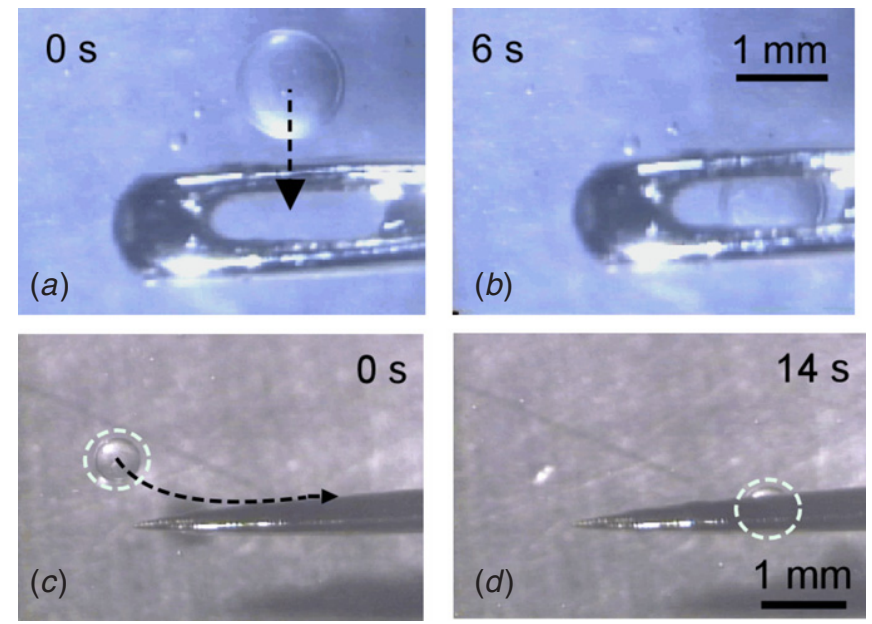

Figure 15. (a), (b) Single droplet trapping with an annular heat flux. Micrographs show the strong affinity of a $1 \mathrm{~mm}$ droplet to the heat source $\left(T \approx 390 \mathrm{~K}\right.$ or $\left.117^{\circ} \mathrm{C}\right) .(c),(d)$ Guidewire pump emulated by a tapered heat flux. Micrographs show the movement of a droplet at $196 \mu \mathrm{m} \mathrm{s}^{-1}$ driven by the suspended heat source $(T \approx 400 \mathrm{~K}$ or $127^{\circ} \mathrm{C}$ ) with $10^{\circ}$ lateral taper and a $5^{\circ}$ taper in vertical spacing. In both experiments, the gap is $\approx 200 \mu \mathrm{m}$.

shaped metal pin $\left(T \approx 390 \mathrm{~K}\right.$ or $\left.117^{\circ} \mathrm{C}, s=600 \mu \mathrm{m}\right)$ was suspended 200-400 $\mu \mathrm{m}$ above the oil layer to approximate an annular heat flux. The micrographs show that a $700 \mu \mathrm{m}$ diameter droplet is actively pulled into the trap, driven by the subsurface flows directed into the center of the trap. If the trap boundary is moved (by the translation of the substrate or heat source), the droplet follows it. The process is repeatable, and the droplet velocity, as it enters the trap, is approximately $200 \mu \mathrm{m} \mathrm{s}^{-1}$. 


\subsection{Tapered heat source}

Experimental confirmation of the guidewire pump (tapered heat source) is shown in figures $15(c)-(d)$. To create the surface heat flux, a steel needle $\left(T \approx 400 \mathrm{~K}\right.$ or $\left.127^{\circ} \mathrm{C}\right)$ with $10^{\circ}$ lateral taper and a $5^{\circ}$ taper in vertical spacing was placed $\approx 200 \mu \mathrm{m}$ above the fluid surface. Time lapse images show a $523 \mu \mathrm{m}$ diameter droplet pulled along the axis by virtue of the Marangoni flows. The droplet moves quickly when first approaching the tip of the heat flux and continues to move in the direction of increasing taper, achieving a maximum velocity of $196 \mu \mathrm{m} \mathrm{s}^{-1}$ at the tip end of the guidewire (i.e., the location of greatest axial temperature gradient).

\section{Discussion}

As mentioned earlier, the approach of generating Marangoni flows by imposing surface temperature gradients confers a number of advantages. The first potential benefit is flow localization: in contrast to isothermal heating, a point or linear heat flux favors the formation of a single Marangoni cell, which allows for precise control of the geometry and flow speeds of the cell. The ability to localize flow is an important aspect as well. The smallest flow cell observed was a $50 \mu \mathrm{m}$ diameter toroidal flow, obtained using a thermal probe above $80 \mu \mathrm{m}$ thick low viscosity oil.

The second benefit is the potential for efficiency: Marangoni flow velocities are proportional to the surface temperature gradient, and the sharp temperature gradients which can be achieved with microscale heat sources enable faster velocities with only small absolute changes in surface temperature. Benefiting from the low thermal conductivity of air (which is nearly an order of magnitude less than oil), the suspended heat sources create sharp lateral temperature gradients at the resolution needed to obtain shaped temperature profiles at the sub-millimeter scale. Embedding heaters in the substrate would diminish these gradients and also waste heat in the substrate.

An additional benefit of not having any physical contact between the heat source and the liquid is that it reduces the likelihood of device contamination and crosscontamination between samples. One of the past approaches to using Marangoni flow for fluidic actuation requires electrochemically generated surfactants [22] which causes ionic contamination of the sample. Temperature gradients generated by substrate heaters [23] or external lasers [24] have also been used; however, both approaches require that the liquid sample be in contact with the substrate. In the present approach, samples are contained in droplets which are suspended in a high-density oil layer, away from the actuator and the substrate below, and they are manipulated entirely using Marangoni flows at the liquid surface. This approach to fluidic manipulation is less prone to contamination than those mentioned above, as well as other popular techniques such as electrowetting $[25,26]$.

With regard to reconfigurability, one of the projected advantages of this type of approach is that the substrate itself remains unpatterned. The complexity and burden of the fluid actuation is essentially placed to the suspended heating structures rather than the fluidic substrate. Since the heating structure can be reused and reconfigured, this can lower the overall cost of repeated experiments.

To achieve the sharpest temperature gradients on the liquid surface, it is important to place the heat sources as close to the surface as possible, since heat spreads isotropically in the air gap. Typical gaps of the order of 100-400 $\mu \mathrm{m}$ can be obtained by visual estimation during the approach of the heat source to the sample surface. An automated technique which would allow for small air gaps would greatly benefit efficiency, as simulations indicate that when the gap is less than $100 \mu \mathrm{m}$, both the surface temperature gradient and resulting velocity sharply increase.

Placing the probes close to the surface also reduces power consumption. Although the low thermal conductivity of air helps achieve a sharp lateral gradient, it impedes heat transfer from the heat source to the liquid surface. Experimental results with the microfabricated thermal probe show that approximately $10 \mathrm{~mW}$ of heat power must be dissipated in order to generate $1 \mathrm{~mm} \mathrm{~s}^{-1}$ flow velocity. Simulations, however, indicate that $<0.2 \mathrm{~mW}$ of surface heat flux is needed to generate the same flow velocity. The discrepancy between the two arises due to the poor heat transfer between the probe and the liquid surface. Three-dimensional heat conduction simulations of the probe above the liquid surface show that at a $100 \mu \mathrm{m}$ separation, the surface temperature rise is only $1.3 \%$ that of the probe. This figure drops to only $0.3 \%$ if the gap is $300 \mu \mathrm{m}$. As shown earlier (figure 4), there is an approximate $1 / x$ dependence on the surface temperature rise versus the probe-liquid separation.

An equally important way to improve efficiency is by optimizing the heat source. Microfabricated heaters with welldesigned thermal isolation can achieve thermal resistances in the range of $10000 \mathrm{~K} \mathrm{~W}^{-1}$ [20] and thermal masses of only $10^{-9} \mathrm{~J} \mathrm{~K}^{-1}$. This enables temperatures of $370 \mathrm{~K}\left(97{ }^{\circ} \mathrm{C}\right)$ with $<10 \mathrm{~mW}$ of input power, and dynamic heating times of the order of $10 \mathrm{~ms}$. In these probes, the relatively small thermal losses are due to conduction through the probe shank. Thus, the efficiency can be further improved by using a thinner cantilever or lower thermal conductivity materials in its fabrication. With the existing devices, the best power efficiency, obtained experimentally at a $30 \mu \mathrm{m}$ gap, is about $1 \mathrm{~mm} \mathrm{~s}^{-1}$ per milliwatt input power, reaching a maximum liquid velocity of $1700 \mu \mathrm{m} \mathrm{s}^{-1}$ with $<20 \mathrm{~mW}$ input power to the probe.

Another purpose of this effort was to show that even simple metal tools can be used to generate Marangoni flow which can emulate microfluidic components. Because these components are larger in size (a few $\mathrm{mm}$ ) than the microfabricated probe $(<100 \mu \mathrm{m})$, they cannot generate as sharp a gradient. Thus, a larger absolute increase in temperature is needed to drive flows at the same rate. In the case of the tapered, ring and linear geometries, the heat source temperatures are between $350-390 \mathrm{~K}\left(77-117^{\circ} \mathrm{C}\right)$, and the liquid surface temperatures are measured to be $10-40 \mathrm{~K}$ above ambient. This temperature requirement can be reduced by scaling the heat sources to smaller sizes. 
Overall, scaling down to smaller length scales can potentially provide significant performance benefits. For example, the microfabricated thermal probe used in this work

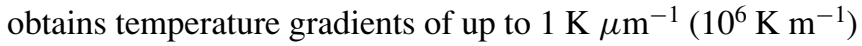
along a $200 \mu \mathrm{m}$ long cantilever. Experimental and simulation data using this probe show that on the liquid surface, the typical temperature gradient ranges from 1 to $10 \mathrm{~K} \mathrm{~mm}^{-1}$, resulting in shear stresses between 0.1 and $1 \mathrm{~N} \mathrm{~m}^{-2}$ and velocities between 1 and $10 \mathrm{~mm} \mathrm{~s}^{-1}$ on a $200 \mu \mathrm{m}$ thick oil layer. Hypothetically, if the thermal gradients on the microfabricated probe (up to $10^{6} \mathrm{~K} \mathrm{~m}^{-1}$ ) could be directly transferred to the fluid surface without loss, this could result in shear stresses of up to $100 \mathrm{~N} \mathrm{~m}^{-2}$ and theoretical velocities above $10 \mathrm{~cm} \mathrm{~s}^{-1}$ (if viscous resistance can be minimized). Although this is not possible in the existing setup, it does illustrate the favorable scaling of this technique and the potential to achieve high speed flow.

\section{Conclusions}

This study has shown that microfluidic Marangoni flows can be engineered through the geometric design of the heat flux. A surprising number of microdroplet operations, including mixing, confining, filtering, trapping and pumping, can be emulated through the creative use of surface and subsurface flows. These are summarized in table 1. As a noncontact technique, such virtual components avoid many of the contamination issues which limit many microfluidic technologies. The future direction of this research is to integrate several of these components into a usable system for droplet manipulation. A system with programmable heat fluxes may be useful for research applications which require flexibility and reusability over portability.

\section{Acknowledgments}

Support was provided in part by the National Science Foundation, the University of Michigan and a Whitaker Foundation Biomedical Engineering Fellowship (ASB). The authors gratefully acknowledge Dr Shamus McNamara for assistance in fabricating the micromachined thermal probes and Seowyuen Yee for assistance in experiments and characterization. Y Gianchandani acknowledges support through the IR/D program while working at the National Science Foundation. The findings do not necessarily reflect the views of the NSF.

\section{References}

[1] Herold K E, Radermacher R and A Klein S 1996 Absorption Chillers and Heat Pumps (Boca Raton, FL: CRC Press)

[2] Adler J 1970 Fluid mechanics of a shallow fuel layer near a burning wick Combust. Sci. Tech. 2 105-13

[3] Koschmieder E L 1993 Bénard Cells and Taylor Vortices (New York: Cambridge University Press)

[4] Bénard H 1900 Les tourbillons cellulaires dans une nappe liquide Rev. Gén. Sci. Pure Appl 11 1261-71

[5] Block J M 1956 Surface tension as the cause of Benard cells and surface deformation in a liquid Nature 178 650-1
[6] Pearson J R A 1958 On convection cells induced by surface tension J. Fluid Mech. 4 489-500

[7] Maroto J A, Pérez-Muñuzuri V and Romero-Cano M S 2007 Introductory analysis of Bénard-Marangoni convection Eur. J. Phys. 28 311-20

[8] Basu A S and Gianchandani Y B 2007 Shaping high-speed marangoni flow in liquid films by microscale perturbations in surface temperature Appl. Phys. Lett. 9003410

[9] Basu A S and Gianchandani Y B 2005 Trapping and manipulation of particles and droplets using micro-toroidal convection currents Proc. Int Conf Solid-State Sensors Actuat Microsyst (Transducers) (Seoul, Korea, June) pp 85-8

[10] Basu A S and Gianchandani Y B 2005 Microthermal techniques for mixing, concentration, and harvesting dna and other microdroplet suspensions Proc. Int. Conf. Miniaturized Systems for Chemistry and Life Sciences ( $\mu T A S)$ (Boston, MA, October) pp 131-4

[11] Basu A S, Yee S Y and Gianchandani Y B 2007 Virtual components for droplet control using marangoni flows: size-selective filters, traps, channels, and pumps Proc. IEEE Int. Conf. Micro Electro Mechanical Systems (MEMS) (Kobe, Japan, January ) pp 401-4

[12] Palit S R 1956 Thermodynamic interpretation of the Eötvös constant Nature 1771180

[13] Eötvös R 1886 Ueber den Zusammenhang der Oberflächenspannung der Flüssigkeiten mit ihrem Molecularvolumen Ann. Phys. 263 448-59

[14] Higuera F J 2000 Steady thermocapillary-buoyant flow in an unbounded liquid layer heated nonuniformly from above Phys. Fluids 12 2186-97

[15] Fluent Corporation 2001 Fluent 6 User's Guide

[16] Taslim M E, Narusawa U and Rifiotis H 1989 Thermocapillary flow induced by a surface heat flux variation ASME Heat Transfer Div. Publ. HTD 113 85-93

[17] Lai C-L and Chai A-T 1986 Surface temperature distribution along a thin liquid layer due to thermocapillary convection Acta Astronaut. 13 655-9

[18] Basu A S 2008 Microthermal devices for fluidic actuation by modulation of surface tension $P h D$ Thesis University of Michigan

[19] Bertrand Y and Hoang L C 2003 Vegetal oils as substitute for mineral oils Proc. 7th Int. Conf. Properties and Applications of Dielectric Materials (Nagoya, Japan, June) pp 491-4

[20] Li M H and Gianchandani Y B 2003 Applications of a low contact force polyimide shank bolometer probe for chemical and biological diagnostics Sensors Actuators A $104236-45$

[21] Song H, Tice J D and Ismagilov R F 2003 Reactions in droplets in microfluidic channels Angew. Chem. Int. Ed. $42768-72$

[22] Gallardo B S, Gupta V K, Eagerton F D, Jong L I, Craig V S, Shah R R and Abbott N L 1999 Electrochemical principles for active control of liquids on submillimeter scales Science 283 57-60

[23] Darhuber A A, Valentino J P, Davis J M, Troian S M and Wagner S 2003 Microfluidic actuation by modulation of surface stresses Appl. Phys. Lett. 82 657-9

[24] Kotz K T, Noble K A and Faris G W 2004 Optical microfluidics Appl. Phys. Lett. 85 2658-60

[25] Cho S K, Moon H and Kim C-J 2003 Creating, transporting, cutting, and merging liquid droplets by electrowetting-based actuation for digital microfluidic circuits J. Microelectromech. Sys. 12 70-80

[26] Yoon J-Y and Garrell R L 2003 Preventing biomolecular adsorption in electrowetting-based biofluidic chips Anal. Chem. 75 5097-102 